\title{
Evaluation of the combination of Rapid Diagnostic Tests and microscopy for imported malaria surveillance in Anhui province, China
}

\section{Weidong Li}

Anhui Provincial CDC: Anhui Provincial Center for Disease Control and Prevention

\section{Xinzhou Zhang}

Anhui Yike Daxue: Anhui Medical University

Jun Feng

National Institute of Parasitic Diseases

Tao Zhang

Anhui Provincial CDC: Anhui Provincial Center for Disease Control and Prevention

\section{Xian Xu}

Anhui Provincial CDC: Anhui Provincial Center for Disease Control and Prevention

Jingjing Jiang

Anhui Provincial CDC: Anhui Provincial Center for Disease Control and Prevention

\section{Shuqi Wang}

Anhui Provincial CDC: Anhui Provincial Center for Disease Control and Prevention

\section{Xiaofeng Lyu}

Anhui Provincial CDC: Anhui Provincial Center for Disease Control and Prevention

Shizhu Li

National Institute of Parasitic Diseases

Manman Lu ( $D$ lumanman123@126.com )

Anhui Medical University

\section{Research Article}

Keywords: imported malaria, rapid diagnostic test, microscopy, Anhui province

Posted Date: December 31st, 2020

DOI: https://doi.org/10.21203/rs.3.rs-136764/v1

License: (c) (1) This work is licensed under a Creative Commons Attribution 4.0 International License.

Read Full License 


\section{Abstract \\ Background}

In the Anhui province, China, efforts to halt local malaria transmission were successful, with no endemic cases reported since 2014. Contrastingly, imported malaria cases are still being reported, indicating a disease reintroduction risk after years of eradication. To avoid this reintroduction, rapid diagnostic tests (RDTs) were combined with microscopy methods to strengthen malaria surveillance. Herein, we aimed to evaluate the efficacy of this surveillance strategy in a field setting.

\section{Methods}

We conducted a retrospective study using malaria surveillance data from January 2016 to June 2020 . Epidemiological characteristics and diagnostic information were analysed using descriptive and comparative statistics. The diagnostic performance of the combined toolbox (RDTs plus microscopy) was evaluated based on its sensitivity, specificity, positive and negative predictive values, and Cohen's kappa coefficient.

\section{Results}

The combined toolbox displayed a higher overall sensitivity for malaria cases than that of microscopy alone $\left(93.74 \%\right.$ vs $\left.89.37 \% ; \chi^{2}=6.09 ; p=0.14\right)$. In contrast to its high sensitivity, the combined toolbox had a specificity of $69.66 \%$. The species identification rates of the combined toolbox for $P$. falciparum, $P$. vivax, P. ovale, and P. malariae were $83.15 \%, 65.00 \%, 42.11 \%$, and $40.00 \%$, respectively.

\section{Conclusions}

The combination of microscopy and RDTs is an effective strategy for malaria surveillance, possibly detecting more $P$. falciparum infections than microscopy alone. However, the specificity and ability to identify species of the combined toolbox were not optimal. Thus, monitoring malaria cases in nonendemic areas may require employing more than one diagnostic tool in surveillance strategies. Moreover, further understanding of the advantages and disadvantages of different detection methods is necessary for applying optimum combinations in the field setting.

\section{Background}

Malaria causes significant morbidity and mortality in humans, representing a major global public health issue. Approximately 228 million malaria cases occurred in 2018, leading to 405,000 deaths globally [. In China, the National Malaria Elimination Action Plan (2010-2020) was launched in 2010 ] with the ambitious goal of eradicating endemic malaria in non-border regions before the end of 2015. Anhui 
province, located in south eastern China, introduced a provincial plan for malaria elimination in that same year. Following the "1-3-7 model" strategy [, local transmission was halted and no autochthonous cases have been reported since 2014 in the province. After maintaining malaria elimination for six years, Anhui province passed the sub-national malaria elimination assessment in November 2019. In contrast, imported malaria cases continue to be reported $[\mathrm{H}$ as people remain generally susceptible to malaria; moreover, Anopheles sinensis is widely distributed throughout the province [. Thus, the region still has a high receptivity and vulnerability to the disease, indicating a reintroduction risk after years of elimination. Therefore, to deploy an effective response to this long-term public health challenge, we must maintain a sensitive surveillance system to rapidly detect imported malaria cases. The low incidence of imported malaria cases led to a lack of microscopists experienced in the disease, representing another challenge to effectively employ microscopy in surveillance strategies [4].

Since 2010, the World Health Organization (WHO) recommends that malaria case management should be based on parasite diagnosis for all suspected malaria cases []. Thereafter, the number of available rapid diagnostic tests (RDTs) and the scale of their use have increased rapidly. RDTs are a vital part of malaria management strategy because it extends access to diagnostic tools in areas where microscopy cannot be reliably maintained. Studies have reported that RDTs were very effective in detecting Plasmodium falciparum (P. falciparum) in various settings in sub-Saharan Africa [, potentially being more cost-effective than either microscopy alone or presumptive diagnosis $\square$. Thus, to improve malaria surveillance quality, RDTs were introduced in Anhui province to provide parasite-based diagnosis in combination with microscopy. Herein, we aimed to evaluate this strategy through a retrospective analysis of malaria surveillance data from January 2016 to June 2020. In addition, we aimed to determine the diagnostic efficacy of RDTs plus microscopy in detecting imported malaria cases in a field setting. Our results may contribute to determining the optimal use of the combined toolbox to prevent malaria reintroduction, as well as providing lessons for other non-endemic areas.

\section{Materials And Methods}

\section{Malaria surveillance system and study population}

Anhui province operates a surveillance system comprising passive case detection (PCD) and active case detection. Of these, PCD is the main surveillance mode. When a patient suspected of having malaria (presenting with fever or other symptoms of malaria combined with a history of travel to a malariaendemic area) seeks medical care, the physician should request a malaria diagnostic test. Microscopy is the routine diagnostic test. Because infections caused by $P$. falciparum, Plasmodium vivax (P. vivax), Plasmodium malariae (P. malariae), and Plasmodium ovale ( $P$. ovale) are still being reported annually [45], two Pf/Pan RDTs (Diagnostic Kit for Malaria, Guangzhou Wondfo Biotech Co., Ltd, Guangzhou, China; and CareStart ${ }^{\text {TM }}$ Malaria Pf/pan Ag Combo RDT, Access Bio, Inc., Somerset, NJ, USA) were introduced to the malaria surveillance strategy. 
The Wondfo RDT detects $P$. falciparum lactate dehydrogenase (Pf-LDH) and Plasmodium lactate dehydrogenase (pan-LDH), while the CareStart RDT detects $P$. falciparum histidine-rich protein 2 (PfHRP2) and pan-LDH. Wondfo tests were distributed to medical institutes, whereas CareStart tests were used as confirmation tests in Centers for Disease Control because they were not registered in the National Medical Products Administration (CFDA). All RDTs used during the study period were purchased and distributed by the Anhui Provincial Center for Disease Control and Prevention (AHCDC). Production lot numbers of Wondfo tests were W05450603WC, W05471202, W05490305, and W05491104; those of CareStart tests were MR15A06, MR17M61, and MR19K66.

All patients who presented to medical centres with suspected malaria between January 2016-June 2020 were included in this study.

\section{Confirmation of malaria cases}

Because of the high risk of malaria, each suspected case is mandatorily reported in China [. When a suspected case is reported through the China Information System for Disease Control and Prevention (CISDCP), the staff of the county Center for Disease Control is notified. Subsequently, the staff collects samples from the patient (whole blood and smears) prior to anti-malarial treatment initiation and records the test results obtained in the medical centre [3]. The samples are sent to the Malaria Diagnostic Reference Laboratory of Anhui Province within 3 days after collection. In the reference laboratory, the final diagnosis is confirmed using microscopic examination and/or real-time polymerase chain reaction (PCR) analysis.

Microscopists conducted microscopic examinations using Giemsa-stained thick and thin blood films at $1,000 \times$ magnification. For the PCR assay, DNA was extracted using the QIAamp DNA mini kit (QIAGEN Inc, Hilden, Germany) according to manufacturer's instructions. Commercial real-time PCR kits (Shanghai ZJ Bio-Tech Co., Ltd, Shanghai, China) were used to distinguish Plasmodium species. The PCR reaction was performed in a 40- $\mu \mathrm{L}$ reaction mixture containing $35 \mu \mathrm{L}$ of reaction mix, $0.4 \mu \mathrm{L}$ of enzyme mix, $1 \mu \mathrm{L}$ of internal control, and $4 \mu \mathrm{L}$ of DNA template. The conditions were as follows: $37^{\circ} \mathrm{C}$ for $2 \mathrm{~min}$ and $94^{\circ} \mathrm{C}$ for $2 \mathrm{~min}$, followed by 40 cycles at $93^{\circ} \mathrm{C}$ for $15 \mathrm{~s}$ and $60^{\circ} \mathrm{C}$ for $60 \mathrm{~s}$. Individuals confirmed to have malaria through microscopy and/or real-time PCR results were defined as malaria cases in this study.

In the reference laboratory, blood samples were tested simultaneously using Wondfo and CareStart RDTs. Both tests were performed according to manufacturers' instructions. Briefly, $5 \mu \mathrm{L}$ of blood was added to the sample well, 2-3 drops of buffer solution were added to the buffer well, and the result was read and recorded after 15 minutes. Both tests have one control line (" $\mathrm{C}$ ") and two detection lines ("T1" and "T2"). T1 and T2 lines indicate $P$. falciparum and Plasmodium infections, respectively. The results are interpreted as follows: $C$ plus T1 lines indicate a positive result for $P$. falciparum infection; $C$ plus T2 lines indicate a positive result for $P$. vivax, $P$. malariae, and/or $P$. ovale infections; all three lines indicate a positive result for $P$. falciparum infection or concurrent infection of $P$. falciparum and other species $(P$. vivax, P. malariae, or P. ovale).

\section{Patient data collection}


Patients' data were collected from the CISDCP database and sample receipt. The data retrieved included age, sex, occupation, and malaria diagnostic test results. The Malaria Diagnostic Reference Laboratory of Anhui Province has professionals responsible for data input and verification.

\section{Data analysis}

The study data were entered into an Excel database (Microsoft Corporation, Redmond, WA, USA), and statistical tests were performed using SPSS version 17.0 (SPSS Inc., Chicago, IL, USA). Categorical data were presented as proportions and percentages. Differences in proportions were compared using Pearson's $\chi^{2}$ test, McNemar's $\chi^{2}$ test, or Fisher's exact test, as appropriate. All statistical tests were twosided and considered statistically significant when $p<0.05$. $P$ values were adjusted for multiple comparisons. Considering the provincial laboratory results as the gold standard, the diagnostic performance of the combined toolbox was evaluated based on the following parameters: sensitivity, specificity, positive (PPV) and negative predictive values (NPV), and Cohen's kappa coefficient. All parameters were calculated with their respective $95 \%$ confidence intervals.

\section{Results}

During the study period, 644 samples of patients suspected of having malaria were tested in the Malaria Diagnostic Reference Laboratory of Anhui Province. Of these, 377 samples tested positive for $P$. falciparum, 76 for $P$. ovale, 20 for $P$. vivax, 20 for $P$. malariae, and 6 for concurrent infections. The remaining 145 cases tested negative for malaria infection. Patients were aged 3-86 years and most were male $(93.94 \%)$. Moreover, $94.41 \%$ of patients reported recently visiting a malaria-endemic country.

\section{Diagnostic performance of rapid diagnostic tests in a laboratory setting}

Considering the provincial reference laboratory results as the gold standard, CareStart tests exhibited sensitivity, specificity, PPV, NPV, and diagnostic accuracy rate of $89.78 \%, 77.93 \%, 93.33 \%, 68.90 \%$, and $87.11 \%$, respectively, whereas those of Wondfo tests were $89.78 \%, 75.17 \%, 92.56 \%, 68.13 \%$, and $86.49 \%$, respectively. CareStart and Wondfo RDTs did not differ significantly in diagnostic performance parameters (Table 1).

[Table 1 here]

Among positive infections, CareStart and Wondfo tests detected $97.08 \%$ and $96.29 \%, 59.02 \%$ and $67.11 \%$, $95.00 \%$ and $90.00 \%$, and $70.00 \%$ and $60.00 \%$ of $P$. falciparum, $P$. ovale, $P$. vivax, and $P$. malariae infections, respectively. They did not differ significantly in ability to detect different malaria pathogen species (Table 2). The sensitivity of T2 line to detect $P$. falciparum was $64.99 \%$ for Wondfo tests and $70.29 \%$ for CareStart tests. Misclassification rates of CareStart and Wondfo RDTs were 1.00\% (5/499) and 1.60\% (8/499), respectively. Specifically, CareStart and Wondfo tests misidentified four and seven cases, 
respectively, of $P$. falciparum infection. Both tests misidentified one case of $P$. vivax infection as a $P$. falciparum infection.

[Table 2 here]

\section{Easiness of use}

Both tests were considered easy to use. Compared with Wondfo tests, CareStart tests have an excellent background clearance and clearly visible test lines. According to manufacturer's instructions, Wondfo test results must be read within 15 minutes. If this period exceeds 30 minutes, the test result is invalidated because abnormal red background interference may occur, obscuring test lines.

\section{Performance of different malaria diagnostic tests in a field setting}

Of 644 cases of suspected malaria in health centres, 640 were tested using Wondfo RDTs and/or microscopy (microscopy: 632 cases; Wondfo RDTs: 563 cases). The remaining four patients received medical care but refused malaria diagnostic tests. Considering the provincial laboratory results as reference, the sensitivity and specificity of microscopy and Wondfo tests were $89.37 \%$ and $93.33 \%$, and $81.82 \%$ and $63.28 \%$, respectively. Moreover, the sensitivity and specificity of the combined toolbox (RDT plus microscopy) were $93.74 \%$ and $69.66 \%$, respectively (Fig. 1). In the field setting, microscopy displayed a higher specificity than that of Wondfo RDTs $\left(81.82 \%\right.$ vs $\left.63.28 \%, \chi^{2}=11.79, p=0.001\right)$. Furthermore, the specificity of Wondfo tests decreased 11.89 percentage points compared with that observed in the laboratory setting $\left(\chi^{2}=41.05, p<0.001\right)$.

[Figure 1 here]

Regarding parasite detection, microscopy alone identified $89.67 \%, 84.21 \%, 95.00 \%$, and $100 \%$ of $P$. falciparum, $P$. ovale, $P$. vivax, and $P$. malariae infections, respectively. Meanwhile, Wondfo RDTs detected $97.55 \%, 75.71 \%, 94.44 \%$, and $86.67 \%$ of $P$. falciparum, $P$. ovale, $P$. vivax, and $P$. malariae infections, respectively. Finally, the combined toolbox identified $94.65 \%$ of $P$. falciparum, $88.16 \%$ of $P$. ovale, $95.00 \%$ of $P$. vivax, and $100.00 \%$ of $P$. malariae infections. Wondfo RDTs had a better ability to detect $P$. falciparum infections than that of microscopy alone $\left(\chi^{2}=17.393, p<0.001\right)$. Conversely, microscopy detected infections caused by other species of pathogens ( $P$. ovale, $P$. vivax, and $P$. malariae) better than Wondfo tests, albeit this assertion lacked statistical evidence (Table 3 ).

[Table 3 here]

Although Wondfo RDTs could differentiate $P$. falciparum from other malaria pathogen species, they were unable to differentiate between $P$. malariae, $P$. ovale, and $P$. vivax infections. Therefore, only microscopy was included in species identification analysis in this study. In the field setting, microscopy displayed pathogen species identification rates for $P$. falciparum, $P$. vivax, $P$. ovale, and $P$. malariae of $83.15 \%$, 
[Table 4 here]

\section{Effect of introducing rapid diagnostic tests in malaria surveillance strategies}

In the field setting, the combination of RDTs and microscopy increased malaria detection sensitivity compared with that of microscopy alone $\left(93.74 \%\right.$ vs $\left.89.37 \%, \chi^{2}=6.09, p=0.14\right)$. During the study period, Wondfo RDTs identified 37 more patients with malaria than microscopy alone. Of these, 34 patients had $P$. falciparum infection and 3 had $P$. ovale infection. In addition, RDTs had 25 cases of false positive results.

\section{Discussion}

The year 2020 marks seven years of successful local malaria elimination in Anhui province. Presently, the priorities in malaria management are two-fold: 1) to determine how to effectively decrease the occurrence of severe or fatal outcomes that are most commonly associated with $P$. falciparum infections [-], and 2) to prevent malaria reintroduction in the region. Historically, $P$. malariae and $P$. ovale parasites were not endemic to Anhui province and, in 1991, endemic $P$. falciparum parasite was successfully eliminated in the region. Currently, the risk of malaria reintroduction is represented by imported cases of $P$. vivax infection, which has a suitable vector (Anopheles sinensis) widely distributed throughout the province [6]. To achieve the aforementioned malaria management priorities, we have focused on improving the surveillance system sensitivity to timely detect imported malaria cases, especially $P$. falciparum and $P$. vivax infections. The introduction and combination of RDTs with microscopy for malaria surveillance is a vital aspect of disease management. Herein, we retrospectively evaluated the effect of this strategy on malaria surveillance.

In this study, we demonstrated that the combined toolbox sensitivity increased over four percentage points compared with that of microscopy alone (93.74\% vs $89.37 \%)$. This improvement in sensitivity was mainly attributed to the excellent RDT sensitivity for detecting $P$. falciparum infections. In the laboratory setting, CareStart and Wondfo tests sensitivities for detecting $P$. falciparum were $97.08 \%$ and $96.29 \%$, respectively. Wondfo tests maintained this level of sensitivity in the field setting. These results are consistent with those reported in previous studies [-]. Because of this improved sensitivity performance, 34 additional $P$. falciparum infection cases were detected during the study period. Furthermore, we evaluated the ability of both Pf/Pan RDTs in detecting other malaria pathogen species. The tests displayed a high efficiency in detecting $P$. vivax infections, whereas the detection rates of $P$. ovale and $P$. malariae infections were not optimal. This difference in detection rates is mainly attributed to the design of current RDTs products, which are principally developed for the detection of $P$. falciparum and $P$. vivax infections, leading to a decreased sensitivity for $P$. ovale and $P$. malariae infections [-]. This indicates that 
more sensitive point-of-care detection methods must be introduced to identify $P$. ovale and $P$. malariae infections in non-endemic areas.

Although the improvement in overall sensitivity for detecting Plasmodium species was statistically significant $\left(\chi^{2}=6.09, p=0.14\right)$, the combined toolbox utility was still underestimated. Medical institutions above the county level were equipped with RDTs distributed by the AHCDC; however, a few high-level hospitals did not use these tests when investigating suspected malaria cases. Specifically, $87.42 \%$ of patients suspected of having malaria were tested with RDTs. This lack of RDT usage has two possible reasons: 1) physicians of high-level hospitals were unaware of the availability of RDTs because they did not consult the hospital management system, and 2) physicians might be sceptical of products provided by other units. We estimate that, if the RDTs were fully utilised, the combined toolbox sensitivity would increase approximately one percentage point. Thus, identifying the reasons for the low utilisation rate of RDTs in high-level hospitals is necessary to improve malaria surveillance sensitivity.

In contrast to its high sensitivity, the combined toolbox displayed an unsatisfactory specificity of $69.66 \%$. This low specificity was mainly attributed to the poor specificity of Wondfo RDTs (63.28\%) in the field setting. Compared with the results in the laboratory setting, the specificity of Wondfo tests decreased 11.89 percentage points in the field setting $\left(\chi^{2}=41.05, p<0.001\right)$. This decrease may have been caused by those administering the RDTs in clinical practice not closely following manufacturer's instructions regarding result reading; if the test results are read after 30 minutes or longer, they may be incorrect or invalidated. Another important factor is positive RDTs results persisting after anti-malarial treatment because HRP2 can be detected in blood samples for up to two weeks after parasite clearance [. A recent report from Demographic and Health Surveys in Africa highlighted the issue of false positive results owing to the prolonged presence of HRP2 in blood after parasite clearance []. Although pLDH degrades faster than HRP2, RDTs could detect the antigen for more than 10 days after anti-malarial treatment []. In contrast, the persistence of mature gametocytes unaffected by artemisinin drugs can produce $\mathrm{LDH}$, leading to a positive RDT result [-]. Thus, the persistence of malaria antigens after treatment may adversely affect RDTs results, increasing their rate of false positive results. In this study, 25 patients who tested negative for malaria infection in microscopic examination but positive with RDTs accepted antimalarial treatment (overtreatment). They were ultimately confirmed as not having malaria infections by the provincial reference laboratory. This indicates that understanding how previous anti-malarial treatment may affect RDT results is important to correctly interpret them.

Regarding pathogen species identification, the combined toolbox had an inferior performance. The RDTs introduced in Anhui province are Pf/Pan combination tests, which can distinguish $P$. falciparum from non-falciparum pathogen species. However, they are unable to further differentiate between $P$. malariae, $P$. ovale, and $P$. vivax infections. Thus, medical centres mainly identified pathogen species using microscopic examination. Species identification rates for $P$. falciparum, $P$. vivax, $P$. ovale, and $P$. malariae were $83.15 \%, 65.00 \%, 42.11 \%$, and $40.00 \%$, respectively. These rates were not optimal. Furthermore, $14.47 \%(11 / 76)$ of $P$. ovale and $25.00 \%$ (5/20) of $P$. malariae infection cases were misidentified as $P$. vivax infection cases. Because of the high risk of malaria reintroduction, misidentifying other Plasmodium 
species infections as $P$. vivax infections may lead to inappropriate disease management strategies. Considering the excellent performance of RDTs in detecting $P$. vivax infections [, county-level Centers for Disease Control should utilise products designed for $P$. vivax detection to address misidentification issues.

Approximately $6 \%$ of malaria cases did not undergo pathogen species identification. Only the results of positive parasite identification were included in our analysis. This lack of identification represented a challenge for choosing appropriate treatment regimens. Furthermore, both RDTs misidentified some $P$. falciparum infection cases as non-falciparum infections. For CareStart tests, this type of false negative result may be caused by the parasite carrying a HRP2 deletion [ and the prozone effect, whereby an excess of antigens (due to high parasitaemia) or antibodies interferes with detection in RDTs [. For Wondfo tests, the reasons for misidentification remain unknown. Although the misclassification rate was low, it may lead to inappropriate treatment regimens. Thus, careful consideration is necessary.

Currently, many types of RDTs are available in the global market. Many Pf/Pan RDTs have a good performance in malaria diagnosis. Moreover, WHO maintains a yearly RDT product testing programme for quality assurance [22] and publishes general guidelines for RDT procurement [. However, only two Pf/Pan tests (Wondfo and BinaxNOW® Malaria) were registered in the CFDA and could, therefore, be used in health facilities. The smaller domestic market may not have attracted the attention of manufacturers, leading to a very limited selection of products.

This retrospective study based on real-world data has two main limitations. Firstly, patients suspected of having malaria were simultaneously tested using microscopy and RDTs. The latter is not only sensitive to $P$. falciparum infections, but it can also distinguish $P$. falciparum pathogens from non-falciparum pathogen species. Thus, the species identification results using microscopy, especially regarding $P$. falciparum detection, may be improved with the inclusion of the results obtained with RDTs. Our previous study supports this hypothesis. In 2018, we conducted a blind recheck of malaria blood sample slides in 170 general hospitals located in Anhui province. The species identification rate for $P$. falciparum was $60.9 \%$ [4]. Secondly, the study population did not include a control comprised of healthy individuals. Many patients reported a recent history of malaria and anti-malarial treatment, amplifying the limitation of RDTs. According to a previous study, questions around the clinical, patient-level, significance of RDTs often arise in non-endemic regions, especially in the context of therapeutic delay [. This is consistent with the results observed in our study. Furthermore, this limitation highlights the importance of training physicians in the application of RDTs, ensuring that they can appropriately interpret their results in combination with epidemiological and clinical contexts.

\section{Conclusions}

A combined toolbox (RDTs plus microscopy) was introduced to provide parasite-based malaria diagnosis in Anhui province. The overall sensitivity of the combined toolbox increased when compared with that of microscopy alone. Thus, this strategy is effective and may aid in detecting more $P$. falciparum infections. 
In contrast, the specificity and ability to identify pathogen species were not optimal. Therefore, the malaria surveillance strategy must be further optimised to address these issues.

We demonstrated that monitoring malaria after disease elimination requires the use of more than one diagnostic tool to strengthen surveillance strategies. Moreover, further understanding the advantages and disadvantages of different malaria detection methods is necessary for employing optimum combinations in the field setting.

\section{Abbreviations}

AHCDC: Anhui Provincial Center for Disease Control and Prevention; CISDCP: China Information System for Disease Control and Prevention; $P$. Malariae: Plasmodium malariae, $P$. ovale: Plasmodium ovale, $P$. vivax. Plasmodium vivax, pan-LDH: Plasmodium lactate dehydrogenase; PCD: passive case detection; PfHRP2: P. falciparum histidine-rich protein 2; Pf-LDH: P. falciparum lactate dehydrogenase; RDTs: Rapid diagnostic tests; WHO: World Health Organization

\section{Declarations}

\section{Ethics approval and consent to participate}

This study was approved by the Institutional Ethics Committee of Anhui Provincial Center for Disease Control and Prevention. As the study was based on a retrospective review of surveillance data, informed consent was waived. Patients' data were anonymised prior to analysis.

\section{Consent for publication}

Not applicable.

\section{Availability of data and materials}

The dataset supporting the conclusions of this article is included as an Additional Table.

\section{Competing interests}

All authors declare that they have no competing interests.

\section{Funding}

This study was supported by the National Science and Technology Key Projects (2016ZX10004222-004) and Key Techniques in Collaborative Prevention and Control of Major Infectious Diseases in the Belt and Road (No. 2018ZX10101002-004).

\section{Authors' contributions}


LW and LM designed the study and guided the methodology; ZX and ZT wrote the first draft; JJ and

WS were responsible for laboratory testing; LX and ZX were responsible for data input and analysis; LS and FJ critically reviewed, discussed, and modified the manuscript. All authors read and approved the final version of the manuscript.

\section{Acknowledgements}

We acknowledge staff members of the city and county Centers for Disease Control and Prevention for their contribution to data collection.

\section{References}

1. World Health Organization. World malaria report 2019. 2019. https://www.who.int/publications/i/item/9789241565721/. Accessed 16 Dec 2020.

2. Feng J, Zhang L, Huang F, Yin JH, Tu H, Xia ZG, et al. Ready for malaria elimination: zero indigenous case reported in the People's Republic of China. Malar J. 2018;17:315.

3. Cao J, Sturrock HJ, Cotter C, Zhou S, Zhou H, Liu Y, et al. Communicating and monitoring surveillance and response activities for malaria elimination: China's "1-3-7" strategy. PLoS Med. 2014;11:e1001642.

4. Li W, Zhang T, Xu X, Jiang JJ, Yu C, Tian CC, et al. Problems Associated with the Diagnosis of Imported Malaria in Anhui Province. Am J Trop Med Hyg. 2020;102:142-46.

5. Zhang T, Xian X, Jiang JJ, Chen X, Tian CC, Qin XX, et al. Risk factors of severe imported malaria in Anhui province. Acta Tropica. 2019;197:104934.

6. Jiang JJ, Zhang T, Xu X, Lü XF, Wang SQ, Tian CC, et al. Surveillance of malaria vectors in Anhui Province from 2016 to 2018. Chin J Schistosomiasis Control. 2020;32:389-92.

7. World Health Organization. Guidelines for the treatment of malaria - Third edition. World Health Organization, Geneva. 2015. https://www.who.int/publications/i/item/9789241549127. Accessed 16 Dec 2020.

8. Boyce MR, O'Meara, Wendy P. Use of malaria RDTs in various health contexts across sub-Saharan Africa: a systematic review. BMC Public Health. 2017;17:470.

9. Ling XX, Jin JJ, Zhu GD, Wang WM, Cao YY, Yang MM, et al. Cost-effectiveness analysis of malaria rapid diagnostic tests: a systematic review. Infect Dis Poverty. 2019;8:104.

10. Wang L, Wang Y, Jin S, Wu Z, Chin DP, Koplan JP, et al. Emergence and control of infectious diseases in China. Lancet. 2008;372:1598-605.

11. Checkley AM, Smith A, Smith V, Blaze M, Bradley D, Chiodini PL, et al. Risk factors for mortality from imported falciparum malaria in the United Kingdom over 20 years: an observational study. BMJ. 2012;344:e2116. 
12. Newman RD. Malaria-Related Deaths among U.S. Travelers, 1963-2001. Ann Intern Med. 2004;141:547-55.

13. Feleke DG, Tarko S, Hadush H. Performance comparison of CareStart ${ }^{\text {TM }}$ HRP2/pLDH combo rapid malaria test with light microscopy in north-western Tigray, Ethiopia: a cross-sectional study. BMC Infect Dis. 2017;17:399.

14. Li W, Zhang T, Jiang JJ, Xu X, Yu C,Zhao L, et al. Evaluation of the effectiveness of a domestically produced rapid diagnostic test (RDT) at diagnosing malaria. Journal of Pathogen Biology. 2013;8:1099-101.

15. Maltha J, Gillet P, Jacobs JJ. Malaria rapid diagnostic tests in endemic settings. Clin Microbiol Infect. 2013;19:399-407.

16. Maltha J, Gillet P, Jacobs JJ. Malaria rapid diagnostic tests in travel medicine. Clin Microbiol Infect. 2013;19:408-15.

17. McMorrow ML, Aidoo M, Kachur SP. Malaria rapid diagnostic tests in elimination settings--can they find the last parasite? Clin Microbiol Infect. 2011;17:1624-31.

18. Florey L. Measures of Malaria Parasitemia Prevalence in National Surveys: Agreement Between Rapid Diagnostic Tests and Microscopy. DHS Analytical Studies; no. 43. 2014. https://dhsprogram.com/publications/publication-as43-analytical-studies.cfm. Accessed 16 Dec 2020.

19. Ursula D, Rohan A, Peter WG, Ewan C. How long do rapid diagnostic tests remain positive after antimalarial treatment? Malar J. 2018;17:228.

20. Bell D, Wongsrichanalai C, Barnwell JW. Ensuring quality and access for malaria diagnosis: how can it be achieved? Nat Rev Microbiol. 2006;4:S7-20.

21. Tjitra E, Suprianto S, Dyer M, Currie BJ, Anstey NM. Field evaluation of the ICT malaria P.f/P.v immunochromatographic test for detection of Plasmodium falciparum and Plasmodium vivax in patients with a presumptive clinical diagnosis of malaria in eastern Indonesia. J Clin Microbiol. 1999;37:2412-7.

22. World Health Organization. Malaria Rapid Diagnostic Test Performance: Results of WHO product testing of malaria RDTs: round 8 (2016-2018). 2018. https://www.who.int/malaria/publications/atoz/9789241514965/en/. Accessed 16 Dec 2020.

23. World Health Organization. False-negative RDT results and implications of new reports of $P$. falciparum histidine-rich protein 2/3 gene deletions. 2016. https://www.who.int/malaria/publications/atoz/information-note-hrp2-based-rdt/en/. Accessed 16 Dec 2020.

24. Mukkala AN, Kwan J, Lau R, Harris D, Kain D, Boggild AK. An Update on Malaria Rapid Diagnostic Tests. Curr Infect Dis Rep. 2018;20:49.

25. World Health Organization. Recommended selection criteria for procurement of malaria rapid diagnostic tests. World Health Organization, Geneva. 2018. https://www.who.int/malaria/publications/atoz/rdt_selection_criteria/en/. Accessed 16 Dec 2020. 
26. Ota-Sullivan K, Blecker-Shelly DL. Use of the rapid BinaxNOW malaria test in a 24-hour laboratory associated with accurate detection and decreased malaria testing turnaround times in a pediatricsetting where malaria is not endemic. J Clin Microbiol. 2013;51:1567-9.

\section{Tables}

Table 1. Diagnostic performance of CareStart and Wondfo rapid diagnostic tests for malaria in a laboratory setting

\begin{tabular}{|lllll|}
\hline Test characteristics & CareStart & Wondfo & $\chi^{2 *}$ & P-value \\
\hline Sensitivity [95\% Cl] & $89.78[87.11-92.45]$ & $89.78[87.11-92.45]$ & 0.000 & 1.000 \\
\hline Specificity [95\% Cl] & $77.93[71.10-84.76]$ & $75.17[68.06-82.29]$ & 0.750 & 0.386 \\
\hline PPV [95\% Cl] & $93.33[91.09-95.57]$ & $92.56[90.22-94.91]$ & 0.219 & 0.706 \\
\hline NPV [95\% Cl] & $68.90[61.74-76.06]$ & $68.13[60.83-75.42]$ & 0.023 & 0.880 \\
\hline Diagnostic accuracy rate [95\% Cl] & $87.11[84.52-89.71]$ & $86.49[83.84-89.14]$ & 0.108 & 0.742 \\
\hline Kappa value [95\% Cl] & $0.65[0.59-0.72]$ & $0.63[0.56-0.70]$ & NA & NA \\
\hline Misclassification rate (\%) & $1.00(5 / 499)$ & $1.60(8 / 499)$ & 0.701 & 0.402 \\
\hline
\end{tabular}

* McNemar's $\chi^{2}$ test

PPV: positive predictive value, NPV: negative predictive value, Cl: confidence interval, NA, not applicable

Table 2. Diagnostic performance of CareStart and Wondfo rapid diagnostic tests (RDTs) for different malaria species in a laboratory setting 


\begin{tabular}{|c|c|c|c|c|c|c|c|c|c|}
\hline \multirow{2}{*}{$\begin{array}{l}\text { Confirmed } \\
\text { results }\end{array}$} & \multirow{2}{*}{$\begin{array}{l}\text { Type of } \\
\text { RDTs }\end{array}$} & \multirow[t]{2}{*}{$N$} & \multicolumn{4}{|c|}{ Results of RDTs $(n)$} & \multirow{2}{*}{$\begin{array}{l}\text { Sensitivity } \\
(\%)\end{array}$} & \multirow{2}{*}{$x^{2 *}$} & \multirow{2}{*}{$\begin{array}{l}\mathrm{P} \text { - } \\
\text { value }\end{array}$} \\
\hline & & & C & $\begin{array}{l}\text { C, } \\
\text { T1 }\end{array}$ & $\begin{array}{l}\mathrm{C}, \\
\text { T2 }\end{array}$ & $\begin{array}{l}\text { C, T1, } \\
\text { T2 }\end{array}$ & & & \\
\hline \multirow[t]{2}{*}{ P. falciparum } & CareStart & 377 & 11 & 101 & 4 & 261 & 97.08 & 0.571 & 0.45 \\
\hline & Wondfo & 377 & 14 & 118 & 7 & 238 & 96.29 & & \\
\hline \multirow[t]{2}{*}{ P. ovale } & CareStart & 76 & 31 & 0 & 45 & 0 & 59.21 & 1.786 & 0.181 \\
\hline & Wondfo & 76 & 25 & 0 & 51 & 0 & 67.11 & & \\
\hline \multirow[t]{2}{*}{$P$ vivax } & CareStart & 20 & 1 & 1 & 18 & 0 & 95.00 & 0 & 1 \\
\hline & Wondfo & 20 & 2 & 1 & 17 & 0 & 90.00 & & \\
\hline \multirow[t]{2}{*}{ P. malariae } & CareStart & 20 & 6 & 0 & 14 & 0 & 70.00 & 0.5 & 0.48 \\
\hline & Wondfo & 20 & 8 & 0 & 12 & 0 & 60.00 & & \\
\hline
\end{tabular}

Bold and italics signify the number of misclassifications

* McNemar's $\chi^{2}$ test

Table 3. Performance of different diagnostic tests in parasite detection in a field setting 


\begin{tabular}{|llllllll|}
\hline $\begin{array}{l}\text { Confirmed } \\
\text { results }\end{array}$ & Type of test & $\boldsymbol{N}$ & Positive & Negative & $\begin{array}{l}\text { Sensitivity } \\
\text { Specificity }\end{array}$ & $\chi^{2}$ & $\begin{array}{l}\text { P- } \\
\text { value }\end{array}$ \\
\hline $\boldsymbol{P . f}$ & Microscopy & 368 & 330 & 38 & 89.67 & Reference & \\
& RDTs & 327 & 319 & 8 & 97.55 & 17.393 & $0.000^{*}$ \\
\hline $\boldsymbol{P . \boldsymbol { o }}$ & Microscopy+RDTs & 374 & 354 & 20 & 94.65 & 6.308 & $0.012^{*}$ \\
& Microscopy & 76 & 64 & 12 & 84.21 & Reference & \\
\hline $\boldsymbol{P .} \boldsymbol{v}$ & RDTs & 70 & 53 & 17 & 75.71 & 1.652 & 0.199 \\
& Microscopy+RDTs & 76 & 67 & 9 & 88.16 & 0.497 & 0.481 \\
\hline & Microscopy & 20 & 19 & 1 & 95.00 & Reference & \\
\hline RDTs $\boldsymbol{m}$ & Microscopy+RDTs & 20 & 19 & 1 & 95.00 & NA & $1.000^{*}$ \\
\hline & Microscopy & 20 & 20 & 0 & 100.00 & Reference & \\
\hline & RDTs & 15 & 13 & 2 & 86.67 & NA & $0.176^{*}$ \\
\hline
\end{tabular}

*Fisher's exact test

P. f. P. falciparum. P. o: P. ovale. P. v: P. vivax, P. m: P. malariae, RDTs: rapid diagnostic tests, NA: not applicable

Table 4. Ability of microscopy to identify different malaria species in a field setting

\begin{tabular}{|c|c|c|c|c|c|c|c|c|c|}
\hline \multirow{2}{*}{$\begin{array}{l}\text { Confirmed } \\
\text { results }\end{array}$} & \multirow[t]{2}{*}{$N$} & \multicolumn{7}{|c|}{ Results of species identification using microscopy } & \multirow{2}{*}{$\begin{array}{l}\text { Species } \\
\text { identification } \\
\text { rate }(\%)\end{array}$} \\
\hline & & P. $f$ & $\begin{array}{l}P . \\
0\end{array}$ & $\begin{array}{l}P . \\
V\end{array}$ & $\begin{array}{l}P . \\
m\end{array}$ & Mixed & Unclassified & Negative & \\
\hline P. $f$ & 368 & 306 & 1 & 5 & 0 & 4 & $\mathrm{x}$ & 38 & 83.15 \\
\hline P. 0 & 76 & 7 & 32 & 11 & 2 & 0 & 12 & 12 & 42.11 \\
\hline$P . V$ & 20 & 3 & 0 & 13 & 2 & 0 & 1 & 1 & 65.00 \\
\hline$P . m$ & 20 & 3 & 1 & 5 & 8 & 0 & 3 & 0 & 40.00 \\
\hline Mixed & 5 & 3 & 1 & 0 & 0 & 0 & 0 & 1 & 0.00 \\
\hline Negative & 143 & 17 & 1 & 0 & 0 & 0 & 8 & 117 & NA \\
\hline
\end{tabular}


Unclassified: species identification was not attempted, and only the results of parasite identification (positive or negative findings) were obtained

\section{Figures}

\section{Image not available with this version}

\section{Figure 1}

Performance of different malaria diagnostic tests in a field setting RDTs: rapid diagnostic tests 